\title{
Bone invasion in secondary syphilis: case reports
}

\author{
J E OLLÉ-GOIG,* J L BARRIO,† M GURGUI,† D MILDVAN* \\ From the *Department of Medicine, Beth Israel Medical Center, New York, USA, and the †Infectious Diseases \\ Unit, Hospital de la Santa Creu i Sant Pau, Barcelona, Spain
}

SUMMARY The affinity of treponemes for bone tissue is well known, but the incidence of bone infection in the early stages of syphilis is uncertain. Although case reports of early bone invasion are few, reviews of large numbers of patients with early syphilis indicate that the incidence is probably greater than at present believed. Two case reports are presented.

Bony infection, particularly of the skull and the long bones of the legs and arms, is a conspicuous characteristic of the tertiary stage of syphilis and of congenital syphilis. Fortunately, these days doctors seldom encounter such extreme cases.

In secondary syphilis, bone invasion is much less obvious, which may lead to the impression that it is rare. A review of published reports showed few that cited it.' Studies of large numbers of patients with primary or secondary syphilis yielded conflicting estimates, perhaps due in part to variations in methods of evaluation. ${ }^{23}$ Syphilis should be suspected, however, whenever a patient's complaint of bone pain in vulnerable areas is accompanied by other relevant symptoms. Overlooking the possibility of syphilis in these cases delays the start of appropriate treatment.

We report on two patients with osteolytic lesions, one of the arms and legs and the other of the skull, who presented in the secondary stage of syphilis.

\section{Case reports}

\section{CASE 1}

A previously healthy woman aged 32 was admitted to hospital because of pain in her legs and forearms. The pain had begun below both knees three months before admission. It was constant, worse at night, not relieved by rest, and had progressed to affect both ankles and forearms. The patient had become anorectic and had lost 18 pounds during the preceding three months. She suffered constant bitemporal headaches. She had recently consulted a doctor for vaginitis, when no lesions were noted on examination. At that time a Venereal Disease Research Laboratory (VDRL) test

Address for reprints: Dr Jaime E Ollé-Goig, Paseo Sta Eulalia 1, 08017 Barcelona, Spain

Accepted for publication 20 September 1987 gave positive results at a titre of $1 / 8$, whereas it had been negative two years before. Because the fluorescent treponemal antibody absorption (FTA-ABS) test result was negative, however, the VDRL test result was thought to be falsely positive. She was given nonsteroidal anti-inflammatory agents. Radiographic evaluations, including scans, were reported to have been negative.

On admission to hospital the patient, a slightly obese, well developed, white woman, was unable to walk. Abnormal physical findings were confined to her legs and arms; the ulnar aspects of both forearms, as well as the tibial aspects of both legs, were extremely tender to touch. Skin overlying the anterior aspect of the tibias was warm, but there was no erythema or swelling. The joints were not inflamed, her lymph nodes were not palpable, and her temperature was normal. Gynaecological examination showed no abnormalities. Laboratory tests showed a white cell count of $5.1 \times 10^{9} / 1$ with $44 \%$ neutrophils, $37 \%$ lymphocytes, $10 \%$ monocytes, and $9 \%$ band forms. Packed cell volume was $38 \%$; erythrocyte sedimentation rate was $43 \mathrm{~mm}$ in the first hour. Urine analysis and blood biochemistry tests all gave results within normal ranges. The rapid plasma reagin (RPR) test gave a positive result at a titre of $1 / 16$, and the FTAABS test result was now positive. Latex fixation gave negative results. The antistreptolysin O (ASLO) titre was 50 TU (Todd units). Throat, urine, and vaginal cultures all proved negative. Lumbar puncture showed normal cerebrospinal fluid that yielded a negative RPR test result. Radiographic examinations of the skull, chest, and limbs yielded normal results.

The patient continued to complain of severe pain. Biopsy of the skin overlying the tender areas of one leg proved to be non-diagnostic. A ${ }^{99 m} \mathrm{Tc}$ (technetium) methylene diphosphonate bone scan, however, showed intensely abnormal uptake in both tibias (figure 1). Repeat radiography 10 days later showed 


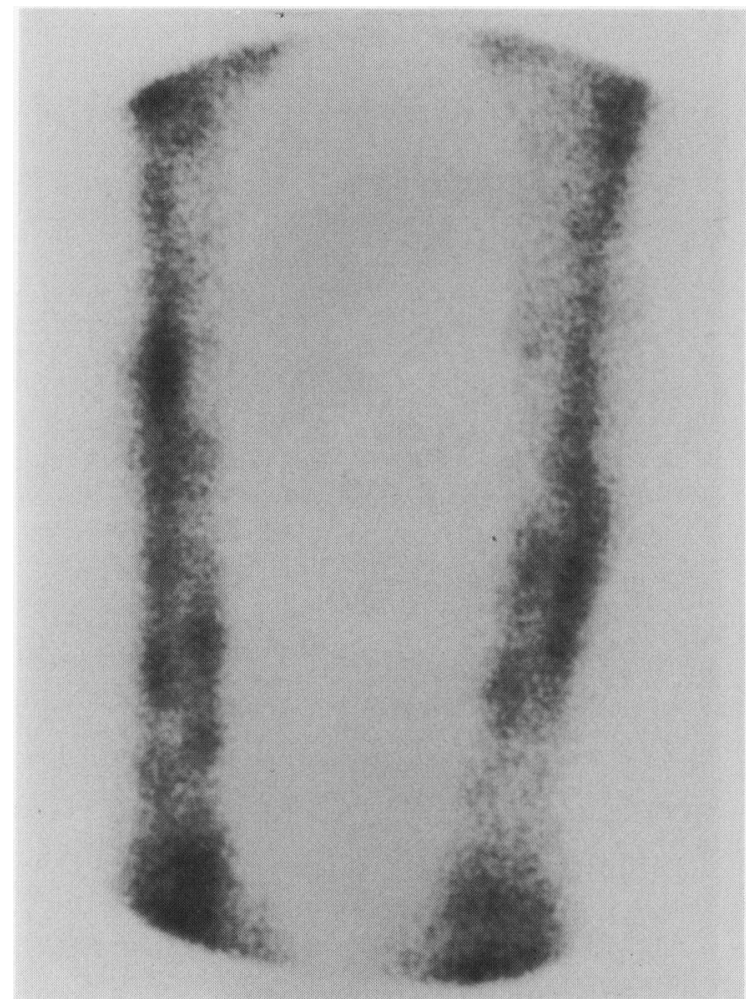

Fig $1{ }^{99^{\mathrm{m}}} T c$ (technetium) radionuclide bone scan showing increased uptake in both tibias.

multiple diffuse intracortical destructive lesions, without sclerosis, in the anterior cortices of the tibias and in the anterior and posterior cortices of the fibulas (figure 2). A localised well organised periosteal reaction was present on the posterior cortex of the lower tibial shafts. A gallium scan showed abnormal uptake in both legs and forearms, which suggested an inflammatory process. Syphilitic osteomyelitis was diagnosed, and the patient was treated with a 10 day course of intravenous aqueous penicillin $G$, followed by a single intramuscular injection of 1.2 MIU benzathine penicillin. At the conclusion of this regimen her pain had almost disappeared and she was able to walk. Her erythrocyte sedimentation rate fell to $18 \mathrm{~mm}$ in the first hour. Six months after discharge, she was symptomless.

\section{CASE 2}

A man aged 21 was referred to us because of a seven week history of asthenia and persistent diffuse headache. He had sustained an injury three months earlier that caused laceration of the skin in the left frontoorbicular area; he had not lost consciousness. He also gave a history of having had gonorrhoea. On admis-

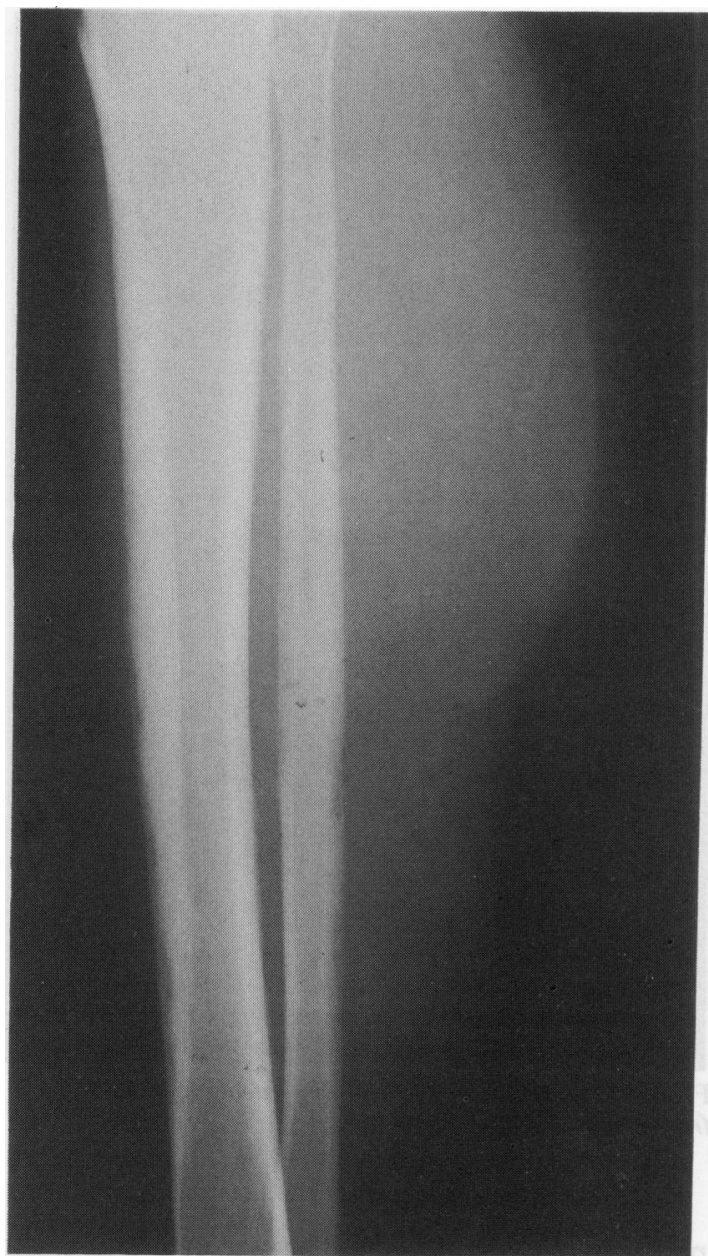

Fig 2 Anterioposterior radiograph of left leg showing lytic lesions in the cortices of tibia and fibula.

sion, his temperature was normal, and the only abnormality noted was painless small multiple adenopathies, which were not fixed, in both sides of the neck and the supraclavicular areas. Routine laboratory test results were within normal limits. Serology tests for cytomegalovirus and Epstein-Barr virus gave negative results. The VDRL test result was negative. A lumbar puncture yielded clear, colourless, acellular cerebrospinal fluid; the protein and glucose concentrations were within normal limits, and the VDRL test result was negative. Radiography of the skull showed an osteolytic lesion in the left frontoparietal area (figure 3). A ${ }^{99^{\mathrm{m}}} \mathrm{Tc}$ methylene dyphosphate scan of the skull showed two areas of abnormally high uptake in the left frontoparietal and supraorbital areas (figure 4). A computed tomogra- 
phic scan of the skull showed an osteolytic lesion with an area of osteitis in the left frontoparietal area.

A bone biopsy of the left frontoparietal area showed necrosis with hypervascularisation and plasma cell infiltrates. Histopathological examination of a biopsy specimen from an enlarged lymph node in the neck showed non-specific reactive adenitis. A generalised, non-confluent, macular rash covering the entire body, including palms and soles, developed two days after the patient had undergone surgery to obtain the biopsy specimens. As this was very suggestive of secondary syphilis, the serum sample obtained on admission was tested again and a prozone phenomenon was shown, with a positive VDRL result at a titre of $1 / 64$. The FTA-ABS test gave a positive result. The patient was treated with 1.2 MIU intramuscular procaine penicillin daily for 10 days and he made an uneventful recovery. When he was seen two months after having completed the above treatment he was asymptomatic and the VDRL test result was positive at a titre of $1 / 8$.

Fig $4{ }^{99^{\mathrm{m}}} T c$ (technetium) radionuclide bone scan showing increased uptake in left supraorbital and frontoparietal areas.

\section{Discussion}

Treponemes have a pronounced affinity for bone tissue. The bone is one of the major systems affected by yaws, a treponematosis found in tropical climates. ${ }^{4}$ The bone may be affected at all stages of syphilis. For several reasons, however, osseous lesions are encountered rarely. ${ }^{5}$ In developed countries, congenital syphilis is now rare, and acquired syphilis is treated promptly. Nevertheless, though congenital syphilis and the gummatous lesions of late tertiary syphilis are rare, bone invasion in the secondary stage is still seen occasionally.

Cutaneous and mucosal lesions are outstanding manifestations of secondary syphilis. ${ }^{6}$ Less common are infections of specific organs as evidenced by hepatitis, meningitis, arthritis, iritis, papillitis, synovitis, myositis, or nephritis. ${ }^{78}$ It is not clear whether bone is seldom affected or whether these lesions are 
not recognised. Few isolated reports have been published, but occasional surveys of patients with secondary syphilis seem to indicate that these lesions are more prevalent than might be anticipated. As in our patients, they can present as a periostitis or osteolytic lesions, or both. The bones most often affected are the skull and the long bones of the limbs-the legs more often than the arms. Principal clinical manifestations are pain in the affected bones, local erythema and increased warmth with tumefaction, headaches, and sometimes generalised symptoms such as weakness, anorexia, and weight loss. ${ }^{19}$

The first description of a case of bone destruction in early syphilis was reported by Lanceraux in France in $1886 .{ }^{10}$ When radiography became available, additional cases were reported. "More recent reports describe invasion of the skull ${ }^{12-16}$ and the limbs. ${ }^{814}$ Shore reported treponemes in the bone biopsy specimen from a patient with lesions in the arms and legs. ${ }^{17}$ Several surveys of large numbers of patients have been published. Wile and Senear reported on 165 patients with primary or secondary syphilis, $36 \%$ of whom had bone or joint invasion. ${ }^{2}$ An even higher percentage might have been found if the patients had been examined radiographically. Frazier and $\mathrm{Li}$ attributed different organ manifestations to racial factors; they found lesions in the bones of $21 \%$ of a series of Chinese men. ${ }^{18}$ On the other hand, a much lower incidence of bone disease was found among 10000 patients with early syphilis studied at Johns Hopkins Hospital during 22 years. ${ }^{3}$ Only $15(0 \cdot 15 \%)$ were found to have destructive lesions of the bone, though only symptomatic patients were radiographed. Thompson undertook an $x$ ray survey of the skulls of 80 patients with secondary syphilis and found that seven $(9 \%)$ had lesions, with destructive osteitis being the most commonly observed. Two of these seven patients had bone disease of their limbs; four reported headaches. ${ }^{19}$

In our first patient, because of an initially negative FTA-ABS test result, the diagnosis of syphilis was not entertained until she was admitted to hospital. At that time, repeat serological testing showed a positive FTA-ABS test result in association with positive results at rising titres in the VDRL. This led to syphilitic osteitis being diagnosed and treated. In the second patient, a prozone phenomenon gave an initial negative VDRL test result, and the correct diagnosis was not entertained until a rash typical of secondary syphilis appeared. A clear understanding of the expected clinical presentation of symptomatic syphilitic osteomyelitis might have permitted an earlier diagnosis in both cases.

In view of the rarity of congenital and tertiary syphilis today, syphilitic osteomyelitis will usually be a manifestation, as in our patients, of secondary syphilis. It is therefore evident that repeated evaluation for early syphilis is warranted in patients similar to ours, who present with generalised symptoms, headache, pain in their limbs, or radiographic indication of periostitis or destructive bony lesions, or both.

$$
\text { 至 }
$$

We thank Dr F Lomeña of the Hospital Clinic i Provincial, Barcelona, who interpreted the bone scans and radiographs.

\section{References}

1 Waugh MA. Bony symptoms in secondary syphilis. British Journal of Venereal Diseases 1976;52:204-5.

2 Wile UJ, Senear FE. A study of the involvement of bones and joints in early syphilis. Am J Med Sci 1916;152:689.

3 Reynolds FW, Wasserman H. Destructive lesions in early syphilis. Arch Intern Med 1942;69:263-76.

4 Yaws. In: Wilcocks CH, Manson-Bahr PEC, eds. Tropical diseases. 17th ed. Baltimore: Williams and Wilkins, 1972:566-79.

5 Jaffe HL. Syphilis of bones and joints. In: Jaffe HL. Metabolic, degenerative and inflammatory diseases of bones and joints. Philadelphia: Lea and Fabiger, 1972:907-51.

6 Tramont EC. Treponema pallidum (syphilis). In: Mandell GL, Douglas RG Jr, Bennett JE, eds. Principles and practice of infectious diseases. New York: John Wiley and Sons, 1985: 1323-33.

7 Parker JDJ. Uncommon complications of early syphilis: hepatitis, periostitis, iritis with papillitis, and meningitis. British Journal of Venereal Diseases 1972;48:32-6.

8 Durston JHJH, Jefferiss FJF. Syphilitic myositis. British Journal of Venereal Diseases 1975;51:141-2.

9 Waugh M. Syphilis as a cause of backache. Br Med J 1972;i:803.

10 Lanceraux E. Les osteites syphilitiques. Annales de Dermatologie et de Syphiligraphie 1886;7:261.

11 Nitchew L. A propos des osteo-periostites syphilitiques precoces. Annales des Maladies Vénériennes 1932;27:600-5.

12 Tauber EB, Goldman L. Syphilitic anemia with diffuse osteitis and superinfection. American Journal of Syphilis and Neurology 1935;19:339-43.

13 Newman BA, Saunders HC. Skeletal system manifestations during secondary syphilis. NY State J Med 1938;38:788-95.

14 Squires JB, Weiner AL. Osteitis in early syphilis: report of a case. Archives of Dermatology and Syphilology 1939;39:830-5.

15 Wile UJ, Welton DG. Early syphilitic osteomyelitis with a report of two cases. American Journal of Syphilis, Gonorrhea and Venereal Diseases 1940;24:1-12.

16 Bauer MF, Caravati CM. Osteolytic lesions in early syphilis. British Journal of Venereal Diseases 1967;43:175-7.

17 Shore RN, Kiesel HA, Bennett HD. Osteolytic lesions in secondary syphilis. Arch Intern Med 1977;137:1465-7.

18 Frazier $\mathrm{CN}$, Li Hung-Chiung. Racial variations in immunity to syphilis. Chicago: University of Chicago Press, 1948.

19 Thompson RG, Preston RH. Lesions of the skull in secondary syphilis. American Journal of Syphilis, Gonorrhea and Venereal Diseases 1952;36:332-41. 